\title{
Fachbeitrag
}

\section{Claudia Lux}

\section{Der Bau der neuen Nationalbibliothek von Katar}

http://doi.org/10.1515/abitech-2018-4002

Zusammenfassung: Die neue Nationalbibliothek von Katar wurde vom niederländischen Architekten Rem Koolhaas entworfen und am 16. April 2018 eröffnet. Die Bibliothek hat nicht nur eine außergewöhnliche äußere Form, sie hat vor allem innen eine Plaza, die über 20000 Quadratmeter groß ist und neben den Terrassen mit Büchern und den Vitrinen in der historischen Sammlung eine Vielzahl anregender Arbeitsplätze und Besprechungsbereiche besitzt. Auch eine Kinderbibliothek und viele neuartige Innovationsräume machen diese Bibliothek zu einem Erlebnis für jedermann.

Schlüsselwörter: Nationalbibliothek, Bau, Katar

\section{The building of the new Qatar National Library}

Abstract: The Dutch architect Rem Koolhaas designed the new Qatar National Library, which opened on April 16, 2018. Not only has the library an exceptional shape, but it also contains a huge 20,000 square meter Plaza with book terraces, a historical collection in glass showcases and many different working spaces and co-working rooms. The children's library and many new innovative spaces make this library an attractive place for everybody.

Keywords: National library, building, Qatar

\section{Das Land}

Katar, ein kleines Emirat im arabischen Golf mit seiner Hauptstadt Doha, ist durch die FIFA Fußball-Weltmeisterschaft, die dort 2022 stattfinden soll, auch in Deutschland bekannt geworden. Das Land, das direkt an Saudi-Arabien grenzt und in den arabischen Golf hineinragt, gilt nach dem Bruttoinlandsprodukt pro Kopf als das reichste Land der Welt. Es hat ungefähr die Größe von Schleswig-Holstein, und von den 2,7 Millionen Einwohnern sind weniger als 300000 Katarer. Die Mehrheit sind Ausländer aus Indien, den Philippinen, Nepal, arabischen und westlichen Ländern, die zumeist für eine begrenzte Zeit in diesem Land leben und arbeiten. Katar wurde erst 1971 von
Großbritannien unabhängig und wird seitdem durch die Erbmonarchie der Al Thani-Familie regiert. Wahlen fanden bisher nur auf kommunaler Ebene statt. Auf der nationalen Eben existiert eine Kammer, in die Personen verschiedener Stämme berufen werden. Der Reichtum des kleinen Landes kommt in erster Linie von den Gasvorkommen, die es im Arabischen Golf besitzt. Insbesondere die frühzeitige Umstellung auf Flüssiggastechnik und LNGTransportschiffe hat Katar zum weltgrößten Flüssiggasexporteur werden lassen. Weniger bekannt ist, wie Katar sich in den letzten Jahren im Bereich von internationalen Sportveranstaltungen, bedeutenden Museen und Universitäten entwickelt hat, mit dem Ziel, seine Wirtschaft zu diversifizieren. Mit der nationalen Vision Katar $2030^{1}$ hat das Land im Jahr 2008 einen Entwicklungsplan hin zu einer Wissensgesellschaft proklamiert. Die Nationalbibliothek ist Teil dieses Prozesses. Im April 2018 konnte nach einer Bauzeit von sechs Jahren der Neubau offiziell eröffnet werden.

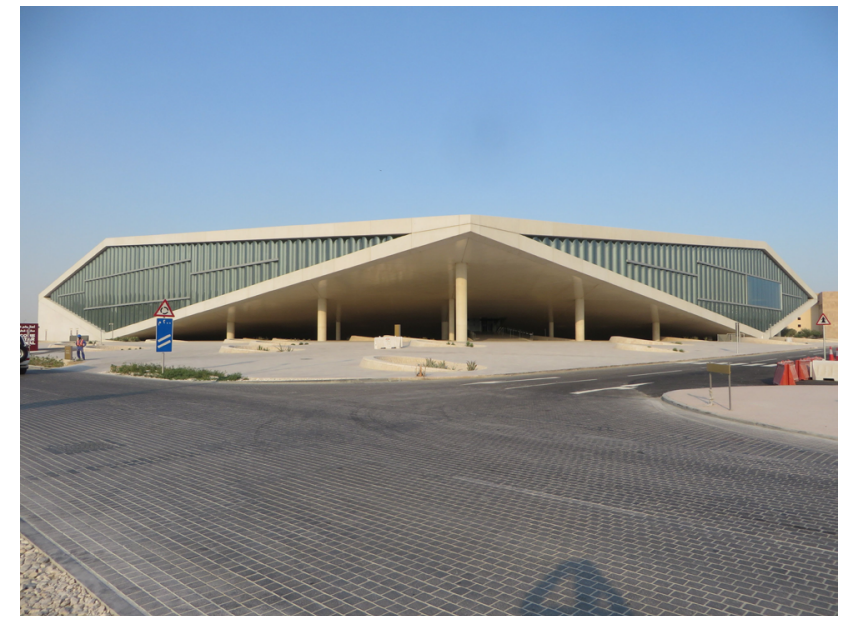

Abb. 1: Nationalbibliothek Katar (Foto: C. Lux)

1 https://www.mdps.gov.qa/en/qnv/Documents/QNV2030_English _v2.pdf (15.09.2018) 


\section{Planungen für eine neue Nationalbibliothek}

Der Bau einer neuen Nationalbibliothek war schon lange überfällig, da die alte Bibliothek Dar-Al-Kutub in einem Gebäude aus dem Jahr 1962 untergebracht war und vor allem die Lagerflächen für das kulturelle Erbe des Landes nicht mehr den notwendigen Standards entsprachen. Es konnte erreicht werden, dass das alte Haus heute unter Denkmalschutz steht, denn es war zu seiner Zeit das erste speziell für eine Bibliothek gebaute Gebäude in der Golfregion, errichtet als eine moderne öffentliche Bibliothek mit Lesesaal und Freihandaufstellung der Bücher. Das Gebäude galt daher auch als Vorbild für die ein Jahr später gegründete erste öffentliche Bibliothek in Abu Dhabi, Hauptstadt der Vereinigten Arabischen Emirate. Erst in den 1970er Jahren, nachdem die UNESCO die Grundlagen für Nationalbibliotheken veröffentlicht hat, begann diese öffentliche Bibliothek in Doha Aufgaben einer Nationalbibliothek, wie die Erstellung der nationalen Bibliographie, zu übernehmen. 1982 erhielt sie per Gesetz das Pflichtexemplarrecht für Veröffentlichungen aus Katar, das inzwischen zur neuen Nationalbibliothek übergegangen ist. Mitte der 1990er Jahre begannen erste Planungen für einen Neubau. Der japanische Architekt Arata Isozaki entwarf 2005 direkt an der Corniche in Doha eine auf drei riesigen Säulen in den Himmel ragende Nationalbibliothek. ${ }^{2}$ Dieses Projekt wurde allerdings kurze Zeit später ad acta gelegt.

Arata Isozaki spielte aber weiterhin eine wichtige Rolle, denn er entwickelte das Gesamtkonzept für die ab 1995 geplante Education City in Katar, ein Projekt der Qatar Foundation, an deren Spitze Scheika Moza bint Nasser, die Frau des damaligen Emirs Scheich Hamad Bin Khalifa Al Thani, steht. In der Bildungsstadt, die sich über fünf Quadratkilometer östlich des Stadtzentrums von Doha erstreckt, wurden ausländische und inländische Universitäten angesiedelt, die mit ihren englischsprachigen Bachelorprogrammen eine neue Generation katarischer Studentinnen und Studenten mit einem international anerkannten Abschluss ausbilden sollen. Dies ist besonders für junge Frauen wichtig, die - wenn sie aus sehr traditionellen Familien kommen - noch nicht alleine im Ausland studieren dürfen. Nach 20 Jahren sind nun in der Bildungsstadt nahezu alle Gebäude errichtet. In dieser vielfältigen Bildungslandschaft lernen heute rund 5000 Studierende an zwanzig Schulen und in acht Universitä-

2 http://www.floornature.de/arata-isozaki-qatar-national-librarydoha-2005-4636/ (15.09.2018). ten, die unter dem Dach der Hamad Bin Khalifa Universität zusammengefasst sind. Inzwischen werden zunehmend auch Masterstudiengänge und Promotionsmöglichkeiten angeboten.

Arata Isozaki entwarf das Vorlesungsgebäude für die Geistes- und Naturwissenschaften sowie das Gebäude der medizinischen Fakultät und ein großartiges Konferenzzentrum. Viele Institutsgebäude ließ er von der mexikanischen Architektengruppe legoretta + legoretta errichten. Der niederländische Architekt Rem Koolhaas erhielt den Auftrag, das Hauptquartier der Qatar Foundation und die neue Nationalbibliothek, die zunächst nur als Universitätsbibliothek geplant war, zu bauen. Nachdem seine Entwürfe fertig waren, bekam die noch nicht gebaute universitäre Zentralbibliothek 2012 die zusätzlichen Aufgaben der Nationalbibliothek und zugleich einer großen öffentlichen Bibliothek zugewiesen und erhielt den Namen Qatar National Library. Auch die dazugehörige U-Bahnstation, die sich noch im Bau befindet, wurde entsprechend umbenannt.

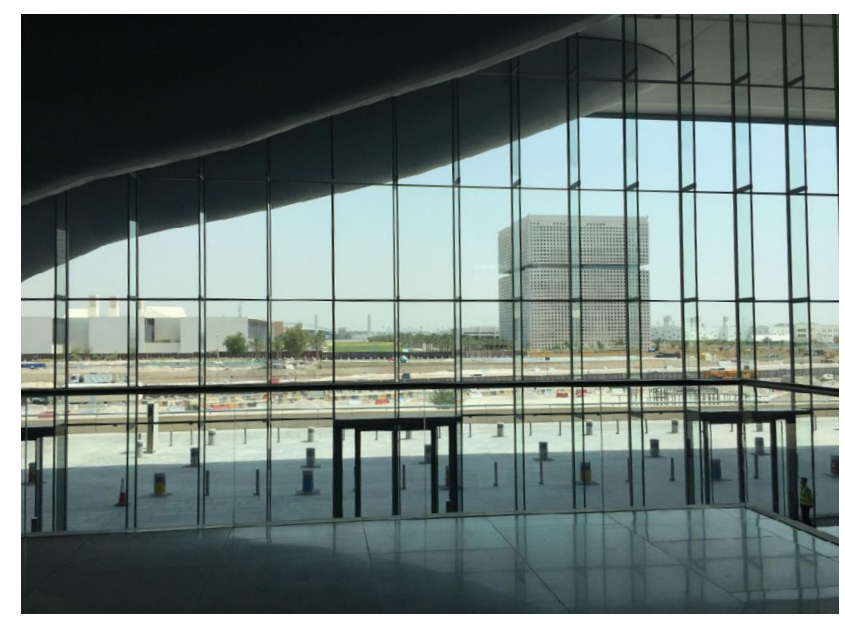

Abb. 2: Blick auf die Bildungsstadt von Aratha Isozakis Konferenzzentrum aus, das hohe Gebäude ist das QF Headquarter, Entwurf Rem Koolhaas (Foto: C. Lux)

Die Nationalbibliothek liegt zentral im Campus der Hamad Bin Khalifa Universität zwischen der medizinischen Fakultät und dem Bereich für internationale Politik, Museumskunde und Bibliothekswissenschaft. Das Studierendenzentrum mit Mensa, Shops, Sport- und Veranstaltungsräumen, sowie die weiteren Fakultäten liegen ganz in der Nähe. Jedes Gebäude in der Bildungsstadt ist für sich Ausdruck einer neuen ästhetischen Architektur und einige haben Architekturpreise gewonnen wie das Institut für islamische Studien mit einer Moschee von Mangera Yvars. ${ }^{3}$

3 http://www.myaa.eu/projects/education-city/ (15.09.2018) 


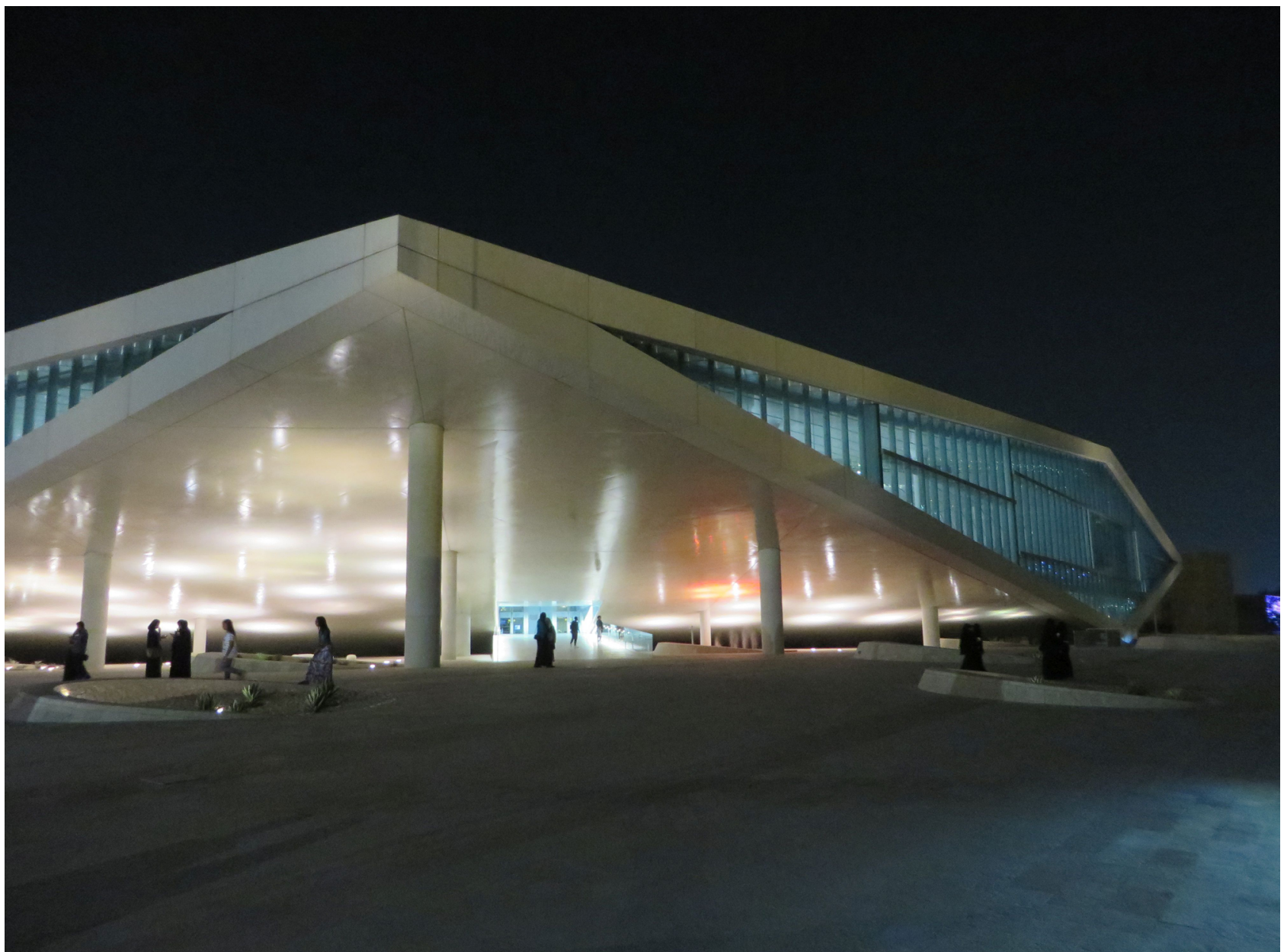

Abb. 3: Nationalbibliothek Katar, Haupteingang am Abend (Foto: C. Lux)

\section{Das Gebäude der Nationalbibliothek}

Das Gebäude der Nationalbibliothek von Katar passt sich von der Höhe her in diese universitäre Landschaft gut ein und besticht durch seine ungewöhnliche äußere Form. Während des Baus wurde häufig danach gefragt, ob hier ein Stadion für die Weltmeisterschaft entsteht, da die wachsenden Terrassen im Innern zu sehen waren. Auch die Größe von ca. 45000 Quadratmetern Nettonutzfläche wirkt beeindruckend, da diese auf nur zwei, teilweise drei Etagen und einer quadratischen Grundfläche von ca. 160 Quadratmetern verteilt und mit einer dem katarischen Klima angepassten Gartengestaltung umgeben sind. Nur von oben zeigt das Gebäude seine quadratische Form. In seiner Erläuterung in einem YouTube-Video ${ }^{4}$ faltet Rem Koolhaas ein Stück Papier, hebt zwei Seiten des Quadrats

4 https://www.youtube.com/watch?v=J44rWRVimc0 (17.09.2018) an und präsentiert bei seinem Entwurf, wo genau er ein Loch in das gefaltete Papier reißt. Dies soll den Haupteingang darstellen, den er fast in der Mitte des Gebäudes platziert. So sollen die Menschen, die in diese Bibliothek eintreten, sofort vom unbegrenzten Wissen der Welt umfangen werden.

Die Außenfassade der Bibliothek ist auf der nördlichen, der Hauptstraße zugewandten Seite rechteckig und zeigt drei Etagen hinter Glas, wobei hier auch das Untergeschoss durch einen offenen Steingarten Licht erhält. Auf dieser Seite befinden sich vor allem Mitarbeiter-, Besprechungs- und Schulungsräume, aber auch die Kinderbibliothek und das Restaurant sowie ein 24/7 Arbeitsbereich. Die drei weiteren nach Osten, Süden und Westen gerichteten Seiten des Gebäudes sind mit speziellem Glas wie langgezogene Rhomben gestaltet. Zwei Ecken des Quadrats sind mit Säulen angehoben, um dem Haupteingang mit seiner leicht ansteigenden Schräge und dem nach unten laufenden Eingang in die Historischen Sammlungen Platz zu bieten. Die blaugraue Fensterfront besteht aus gerunde- 
ten, UV-Strahlen abweisenden Bauteilen. Das Glas hält die Sonne ab und lässt das Licht hinein. Die Fenster wirken am Tage kühl, und verwandeln die Bibliothek nachts in ein wunderbar glänzendes, von innen heraus strahlendes Objekt.

\section{Plaza der Bibliothek, Terrassen und Brưcke}

Im Innern dringt das Licht durch das beschichtete, oval geformte Glas und durch langgestreckte Lichtspalten in der Decke, in deren glänzendem Weiß sich das Licht noch einmal besonders spiegelt und diffus verteilt, so dass nahezu überall gutes Tageslicht in der Bibliothek vorherrscht. Auch der Fußboden aus hellem CarraraMarmor trägt zu diesem Eindruck bei. Wenn es draußen dunkel ist, strahlen die LED-Lichter der silberweißen Metallregale, werden in der Decke widergespiegelt und vermitteln eine besonders glanzvolle Atmosphäre. An drei Seiten bilden Terrassen aus grauem Marmor die Basis für die Bücherregale, dazwischen sind verschieden gestaltete Lese- und Arbeitsplätze sowie gemütliche Sofaecken platziert. Entlang der Terrassen direkt vor der Fensterfront ermöglicht ein sogenannter „people mover“, ein schrägfahrender Aufzug, dass in der Mobilität eingeschränkte Menschen auf alle Ebenen gelangen, um den Bestand zu nutzen. Zusätzlich gibt es begehbare Schrägen mit Glasgeländer, um mit dem Bücherwagen schnell auf andere Ebenen fahren zu können.

Während die Plaza insgesamt eine Fläche von 13459 Quadratmetern umspannt, nehmen die Terrassen mit den Buchregalen eine zusätzliche Nutzfläche von 5177 Quadratmetern ein, so dass zusammen mit der Brücke ein offener Raum von 20223 Quadratmetern den Gesamteindruck der Nationalbibliothek von Katar bestimmt. 400000 Bücher bringen Farbe in den Freihandbereich, weitere 800000 Medien können im Untergeschoß in Kompaktanlagen aufbewahrt werden. Auf 3214 Quadratmetern stehen hier ca. 30000 Regalmeter Stellfläche zur Verfügung. Inzwischen befinden sich 910000 Medien im physischen Bestand der Bibliothek, deren wissenschaftlicher Teil nach den Studienangeboten in der Bildungsstadt und den Forschungsbereichen im Land zusammengestellt wurde.

Hauptsächlich englischsprachig sind hier die wissenschaftlichen Werke der letzten fünf Jahre vorhanden. Teilweise wurden Sammlungen hinzugekauft. Arabischsprachige Medien wurden zunächst auf Buchmessen, später auch über Verlage, die zugleich die Katalogisierung der
Medien anboten, beschafft. Die fachliche Auswahl anhand von Listen lag immer in den Händen der Bibliothekare der Nationalbibliothek. Dies gilt auch für die populärwissenschaftlichen Medien, deren englischsprachiger Teil ebenfalls regalfertig eingekauft wurde. Erst kurz vor der Probeöffnung wurden Zeitungen, CDs und DVDs beschafft. Diese sind in speziell dafür gestalteten Regalen auf der Ostterrasse untergebracht. Daneben liegt auch der Bereich der Jugendbibliothek, vor dem elf große Bildschirmpaneele stehen, auf denen interaktive Spiele und Informationen präsentiert werden. Ein spezieller Gaming-Bereich ist hier ebenfalls auf der Freifläche angesiedelt. Die sachliche Ordnung des Bestandes im Freihandbereich ist in den Regalen beginnend an der Südwestterrasse von unten nach oben entsprechend der Klassifikation der Library of Congress (LoC) gehalten. Nur die Jugendbibliothek und die Kinderbibliothek weichen davon ab, da hier die Dewey Dezimalklassifikation, die in Katar aus den Schulbibliotheken bekannt ist, angewandt wird.

Der offene Raum der Bibliothek wird von einer Brücke überspannt, die 20 Meter breit und mehr als 120 Meter lang ist. 1587 Quadratmeter stehen auf dieser Brücke als Arbeits- und Aufenthaltsfläche zur Verfügung. Neun elektronisch buchbare mit Bildschirmen ausgestattete Gruppenlernräume à 25 Quadratmeter und ein großer Vortragssaal mit Übersetzerkabinen, ein Ausstellungsbereich mit Vitrinen und erhöhte Reihenarbeitsplätze mit Barhockern bieten unterschiedliche Arbeits- und Unterhaltungsmöglichkeiten. Ein Teil der Brückenumrandung ist durchsichtig, so dass man von der Brücke direkt in die Historische Sammlung blicken kann.

\section{Die historische Sammlung, Digitalisierung und Bestandserhaltung}

Von oben wirkt die historische Sammlung wie eine typische antike Ausgrabungsstätte des Vorderen Orients. Sie ist eindeutig das Herz der neuen Bibliothek und beherbergt die historische Sammlung, die ca. 71000 Sammlungsgegenstände zumeist von vor 1950 beherbergt. Das älteste Werk stammt aus dem 7. Jahrhundert. Auch von der Plaza aus kann man auf die in die Mauern eingelassenen Regale mit ihren wertvollen Beständen blicken. Zwei Balkone bieten einen noch besseren Blick auf die Präsentation der Schätze.

In einem gläsernen Fahrstuhl fährt man von der Brücke hinunter, oder man nimmt die Treppe, die von der 


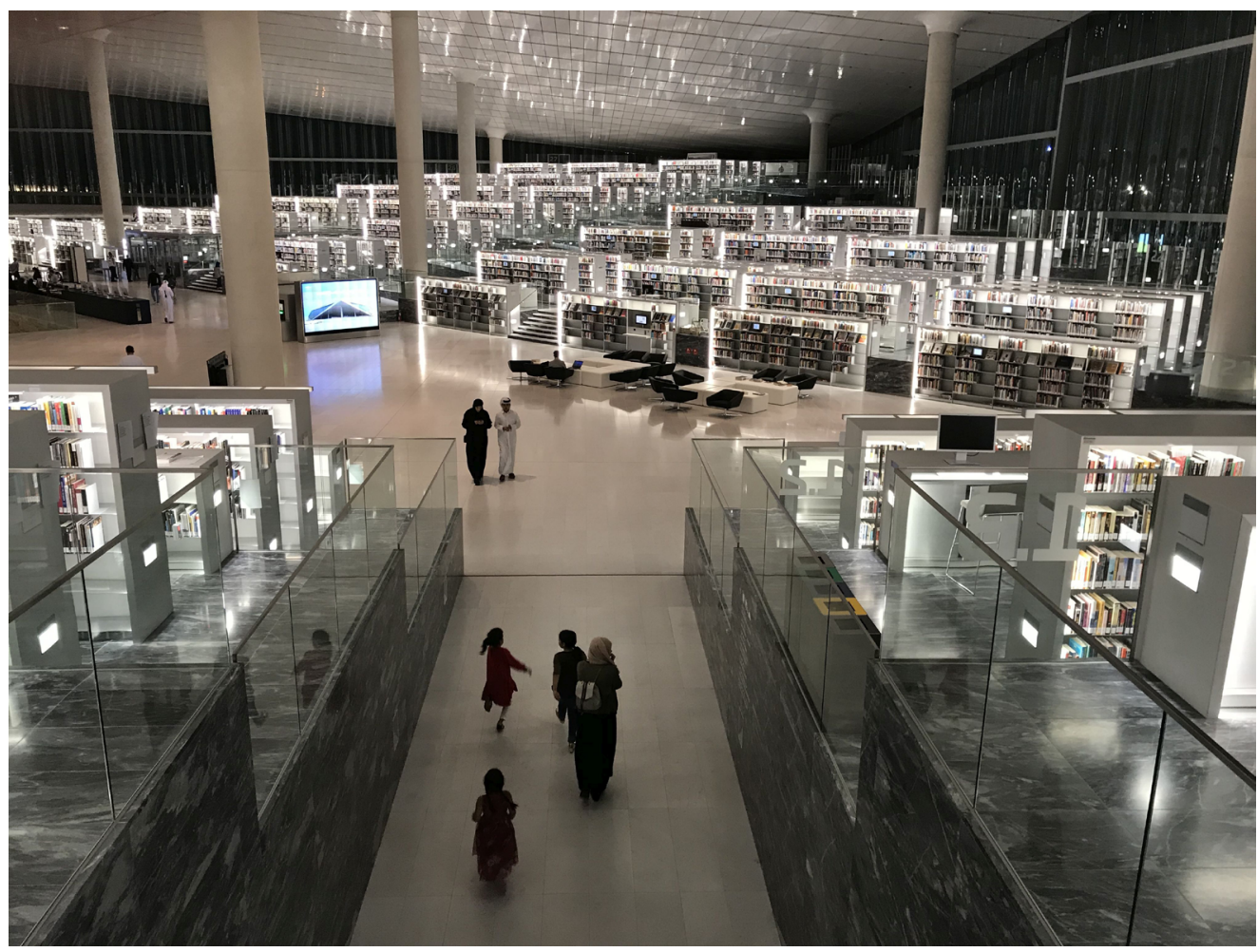

Abb. 4: Nationalbibliothek Katar, Terrassen mit dem Buchbestand (Foto: C. Lux)

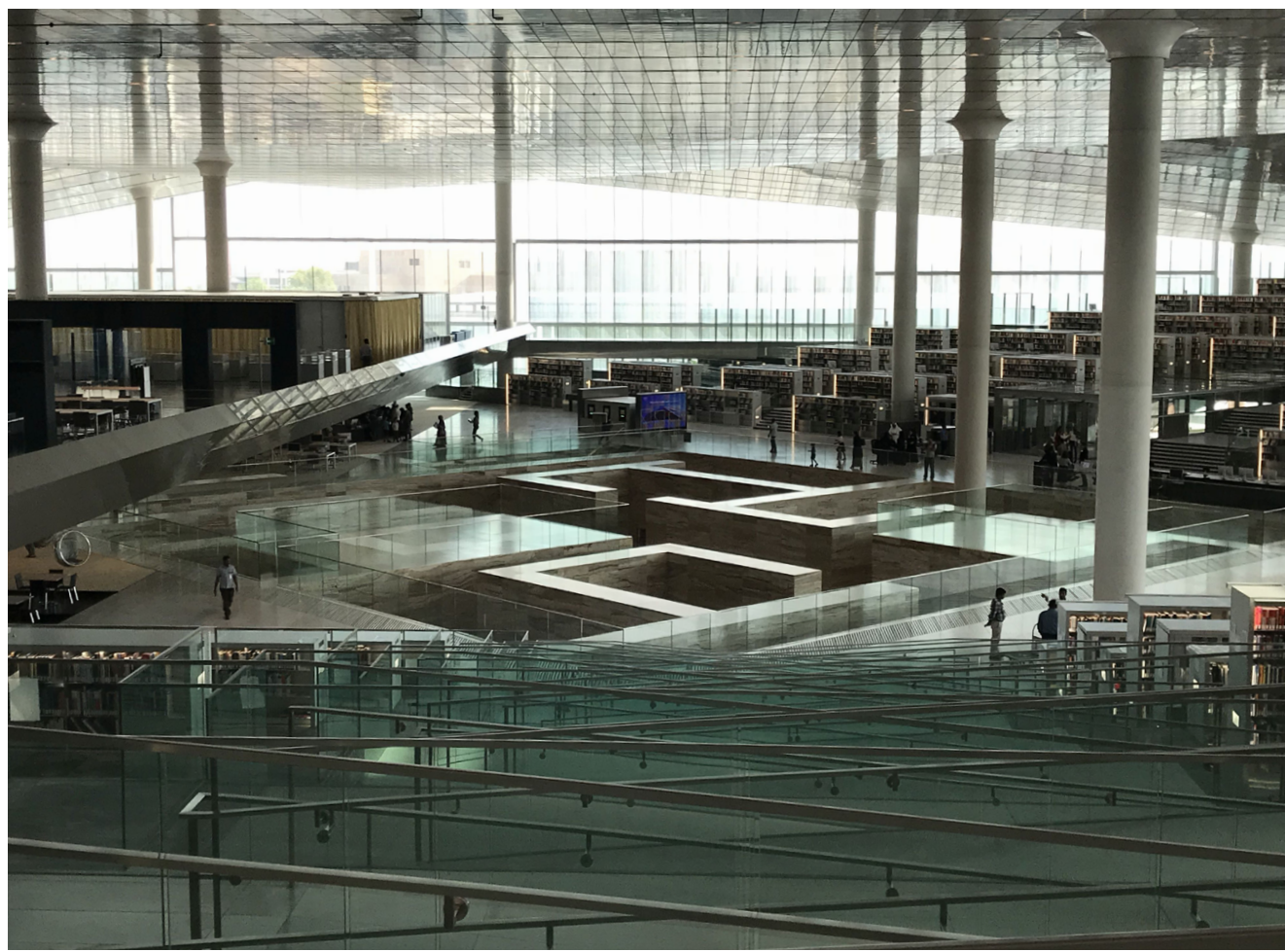

Abb. 5: Nationalbibliothek Katar, Blick auf die Plaza mit Brücke und Historischer Sammlung (Foto: C. Lux) 


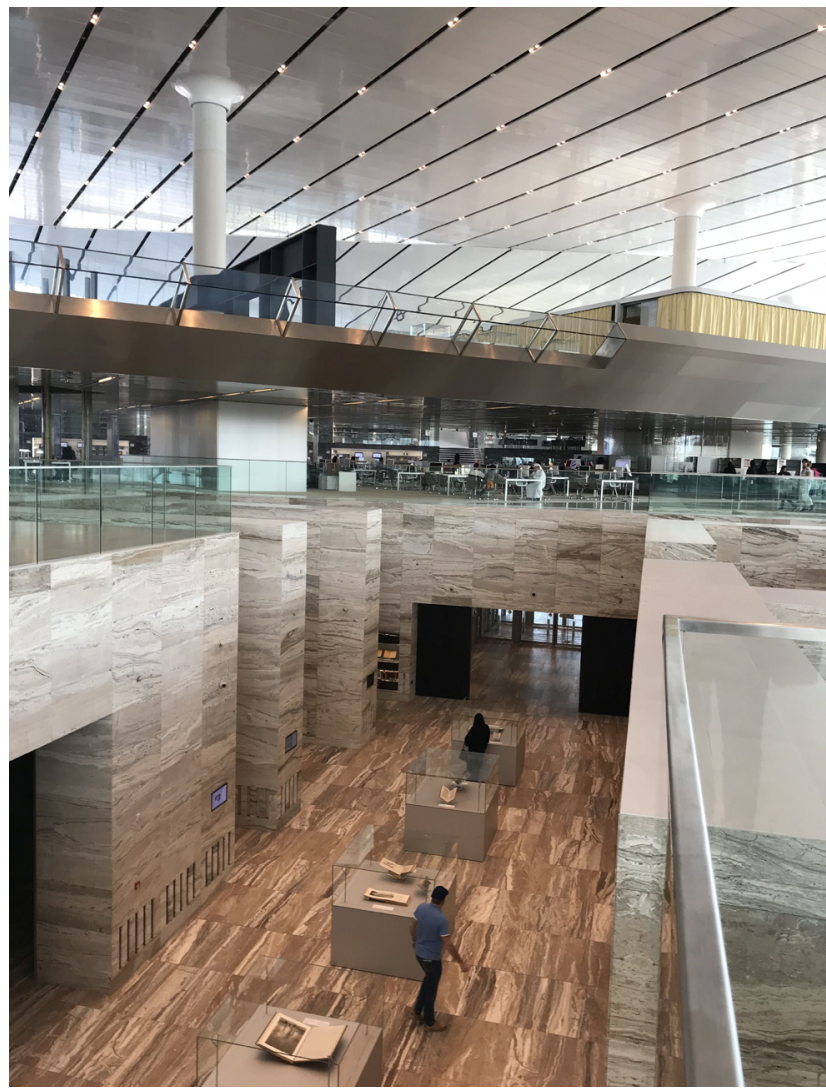

Abb. 6: Nationalbibliothek Katar, Ausstellung der Historischen Sammlung (Foto: C. Lux)

Plaza aus hinunterführt. Hier verändert sich der weiße Marmor der Plaza und man ist umgeben von edlem grauweißem Travertin, der den gesamten Bereich der historischen Sammlung charakterisiert und ihm ein antikes Flair beschert. Die Farbgebung des Travertins ändert sich mit dem Sonneneinfall und wirkt mal kälter, mal wärmer. Der Ausstellungsbereich der historischen Sammlung ist mit einem Schiebetor aus dunklem Holz verschlossen, auf dem in Gold das arabische Wort „iqra“ steht: Das heißt „Lies!“ und ist der Beginn der ersten Offenbarung an Muhammad. Wenn sich das Tor öffnet, meist ist es geöffnet, blickt man auf eine Reihe von Vitrinen, in denen wertvolle Handschriften, Bücher und Karten der Nationalbibliothek präsentiert werden. Rechts und links von diesem mittleren Gang gehen offene Bereiche ab, in denen hinter Glas verschiedene Themen der historischen Sammlungen präsentiert werden, denn die Sammlung ist eine der wertvollsten und relevantesten in der Region. So findet man unter anderem Bücher und Manuskripte zum Thema Katar, darunter sind einige Bücher, die ein Emir vor einem halben Jahrhundert hat drucken lassen.

Manuskripte der arabischen und islamischen frühen Wissenschaften, teilweise in Latein, verdeutlichen die
Verbreitung und den Einfluss der arabischen Welt bis nach Europa - von der Medizin bis zur Algebra. Historische Fotografien und Karten wie auch Reiseberichte über die arabische Welt bieten einen besonders anschaulichen Geschichtsunterricht. Weitere Themen sind arabische Frauen und die heiligen religiösen Stätten. Nicht alle wertvollen Werke der historischen Sammlungen können gleichzeitig präsentiert werden, so dass die Ausstellung immer wieder erneuert werden kann. Die Vitrinen sind klimakontrolliert, mit speziellen Lampen versehen und von sehr hoher Schutzqualität, damit die ausgestellten Objekte keinen Schaden nehmen.

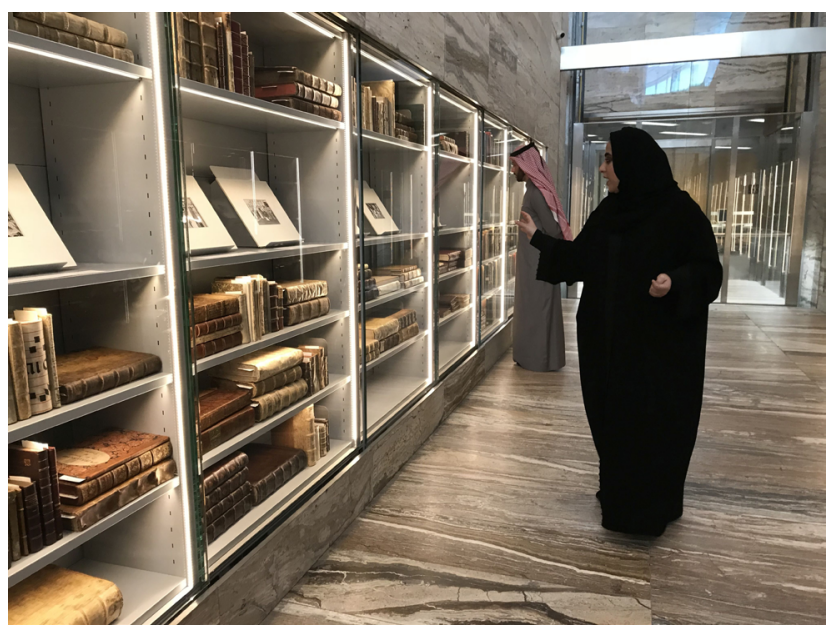

Abb. 7: Nationalbibliothek Katar, Führung in der Historischen Sammlung (Foto: C. Lux)

Aufgrund der teilweise extremen Wetterlage in Katar kontrolliert die Abteilung Bestandserhaltung regelmäßig Temperatur, Luftfeuchtigkeit und Bio-Beschädigungen durch Insekten oder Schimmelsporen im gesamten Gebäude mit der entsprechenden Technik. Auch wenn das Gebäude insgesamt klimatisiert ist, können dennoch Probleme auftreten. In enger Zusammenarbeit mit dem Gebäudemanagement werden die Schwachstellen geschlossen. Die Bibliothek besitzt auf 225 Quadratmetern ein eigenes Labor, in dem alle notwendigen technischen Geräte und Methoden für eine moderne Bestandserhaltung und Restaurierung vorgehalten werden und in dem auch schwer beschädigte Manuskripte untersucht und restauriert werden können. Seit 2014 ist die Abteilung Bestandserhaltung und Restaurierung der Nationalbibliothek von Katar das IFLA Preservation and Conservation Center (PAC) für die arabischen Staaten und arbeitet dabei auch mit dem Memory of the World Program der UNESCO zusammen. Dafür erstellen die Mitarbeiter und Mitarbeiterinnen Schadensaufnahmen und Schadensberichte, geben einen Über- 


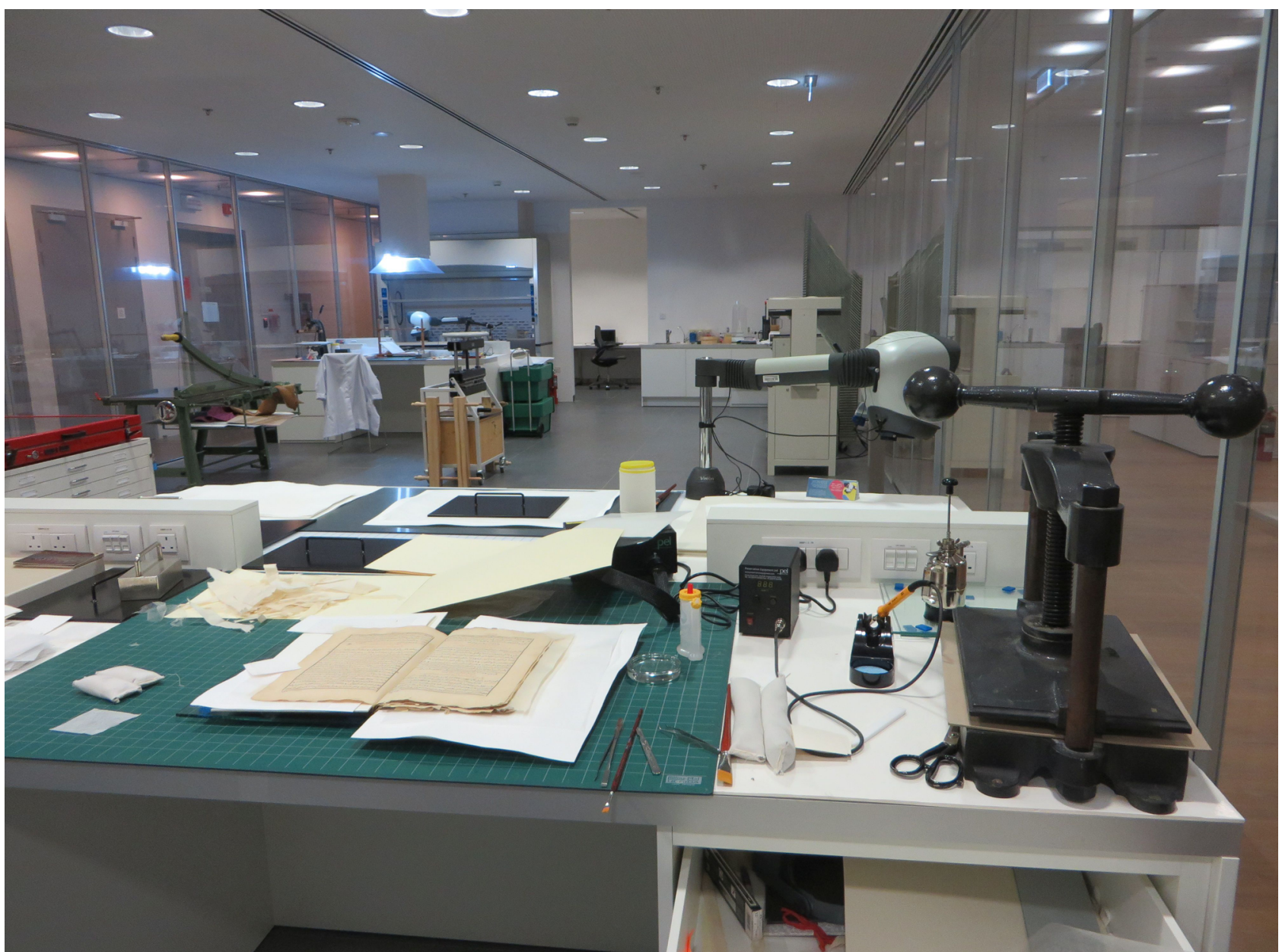

Abb. 8: Nationalbibliothek Katar, Restaurierungswerkstatt (Foto: C. Lux)

blick über die modernen Methoden der Bestandserhaltung und bieten Fortbildungen zu den genannten Themen an. Immer wieder muss vor allem in den arabischen Staaten auf die Gefahren durch Klimaeinflüsse und Insekten, aber auch durch Krieg und Zerstörung hingewiesen werden, um Schutzmaßnahmen rechtzeitig einzuleiten.

Gleich neben der Historischen Sammlung befindet sich im Untergeschoß der Nationalbibliothek auch das Digitalisierungszentrum, das auf einer Größe von 307 Quadratmetern mit einem speziellen Fotostudio neu aufgebaut wurde. Hier werden Rara, Karten, Manuskripte, Fotografien und anderes digitalisiert. Es kann sowohl Massendigitalisierung wie auch Großformat- und Einzeldigitalisierung durchgeführt werden. Das Digitalisierungszentrum bietet seine Dienstleistungen auch anderen Einrichtungen, vor allem Museen und Privatsammlern, an. Bei jeder Maßnahme wird untersucht, welche Art der Digitalisierung für das bestehende Objekt am geeignetsten ist. Für spezielle Rara und brüchige Materialien wird ein besonderes Gerät, der Traveller's Conservation Copy
Stand, genutzt. Alle digitalen Bilder werden hinsichtlich der Farbtreue durch systematische Bildbearbeitung optimiert und die Texte werden mit OCR für lateinische oder arabische Schrift bearbeitet, um sie digital recherchierbar zu machen. Auch 3D-Fotografie wird im Fotostudio angeboten, um Objekte, wie z. B. Globen, virtuell darstellen zu können. Zusätzlich kümmert man sich hier um die Langzeitarchivierung, um durch hohe Standards die Langlebigkeit der digitalen Formate zu garantieren.

\section{Die Informationstechnik, die Buchtransportanlage und die RFID Technik}

Im Untergeschoß befinden sich auch die Räume für die Mitarbeiterinnen und Mitarbeiter der Informationstechnologie, die das Bibliothekssystem und die Webseiten der Bibliothek sowie die notwendige Technik verwalten. In 
der Nationalbibliothek wird gegenwärtig das Bibliothekssystem Sierra von Innovative Interfaces Incorporated (III) eingesetzt. Auch ein eigenes Stromversorgungssystem für den Notfall ist im Neubau vorhanden, so dass bei einem möglichen Stromausfall das System der Nationalbibliothek weiter stabil laufen kann. Generell wurde beim Bau besonders darauf geachtet, den Sicherheitsvorschriften und allen möglichen Notfallsituationen gerecht $\mathrm{zu}$ werden. Für die Feuersicherheit konnte man wegen des offenen Gebäudes und der großen Räume nur eine Sprinkleranlage installieren. Alles wird per Computer überwacht und geregelt. Natürlich ist auch die Türsicherheit der einzelnen Räume elektronisch geregelt, so dass keine Schlüssel ausgegeben werden, sondern die berechtigten Bereiche mittels einer elektronischen Schließanlage öffnen und schließen.

Am Rande der Kompaktanlagenbereichs gibt es noch weitere 27 Studierkabinen, die zu einem späteren Zeitpunkt genauso wie das gesamte Magazin für die Nutzer freigegeben werden können. Dazu müssten aber für das Personal neue Bereiche in der Nähe der Bibliothek geschaffen werden, denn die Planung der alten Zentralbibliothek für die Universität war nicht von dem jetzt notwendigen Personalstamm von geplanten 200 Personen ausgegangen.

Vor dem Eingang zum Magazin im Untergeschoß befindet sich die Verteilstelle für die Buchtransportanlage, an die auch die Rückgabeautomaten in der Plaza und auf der Straße vor dem Eingang der Bibliothek angeschlossen sind. Es gibt 29 Verteilstellen auf den Terrassen, zu denen die Bücher transportiert werden.

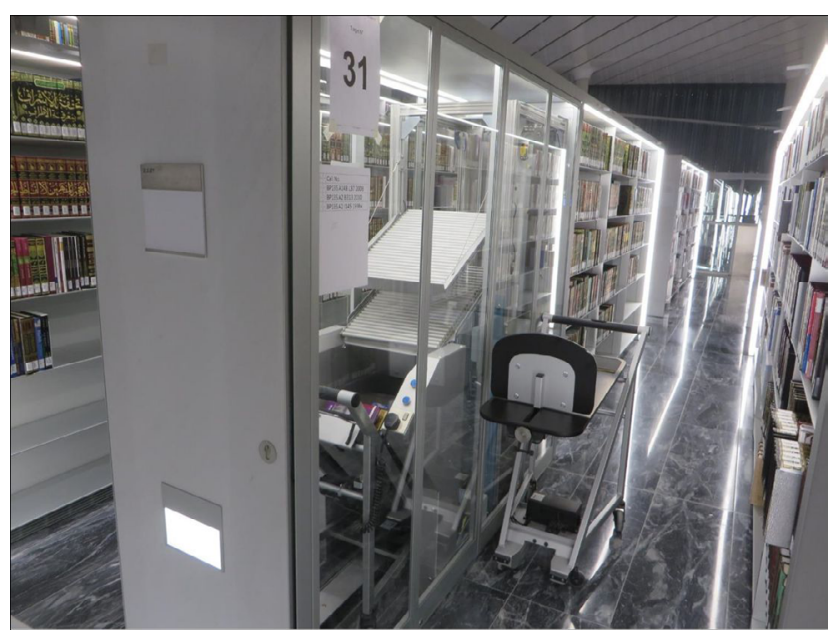

Abb. 9: Nationalbibliothek Katar, Buchtransportanlage und Bücherwagen (Foto: C. Lux)
Die Transportanlage stammt von der Firma Gilgen aus der Schweiz. Es handelt sich um ein offenes Fließbandsystem, auf dem die Bücher einzeln lagern und sich an den Endpunkten in speziell gestalteten Bücherwagen stapeln. Diese werden dann vom Personal zum Einstellen der Medien genutzt. Das damit verknüpfte RFID-System von Biblioteca versorgt auch die Sicherheitsschleuse an den Ausgängen und steuert den gesamten Prozess der Selbstbedienung bei der Ausleihe und der Rückgabe. Die Ausleihgeräte sind in der gesamten Bibliothek verteilt, während die Rückgabeautomaten speziell im Eingangsbereich zu finden sind. Rechts unter der die Bibliothek überspannenden Brücke befindet sich der Bereich, in dem die Bibliotheksausweise ausgestellt werden, über das Internet reservierte Bücher bereitliegen und wo man sich über die Benutzungsbedingungen informieren kann. Dahinter bewegt sich auf einem Laufband, ähnlich wie bei einer Sushi-Bar, das Angebot der neuesten Erwerbungen vorbei, an dem man sich auch direkt „bedienen“ kann.

\section{Arbeitsplåtze, Kinderbibliothek und 24/7 Zone}

Insgesamt stehen 1052 Arbeits- und Veranstaltungsplätze, 528 weiche Sitzmöglichkeiten in unterschiedlichen Formen als Sitzkissen, Sofa oder Sessel für die Besucher zur Verfügung.

Besonders beliebt sind die Arbeitsplätze zur Katalogrecherche und Internetnutzung direkt neben der Benutzungstheke, da man von hier einen wunderbaren Blick über die Plaza hat und sieht, was in der Bibliothek geschieht. Ganz oben auf den Terrassen an der Nordseite befindet sich eine Art katarisches Madschlis, ein Raum mit bequemen Sitzkissen. Von hier aus hat man, sofern man steht, nicht nur eine fantastische Sicht in den Bibliotheksraum, sondern kann auch mittels entsprechender Hardware das neue Rauminformationssystem nutzen. Ein gleiches Gerät steht auch noch einmal im Eingangsbereich.

Andere Nutzer und Nutzerinnen sitzen lieber in den frei hängenden Schaukelsitzen direkt vor dem Lesecafé.

Dahinter befindet sich umgeben von einem wunderbar gestalteten Vorhang, der zugleich als Schallschutz dient, ein offenes Theater für kleine und größere Veranstaltungen. Hier finden Konzerte, Filmvorführungen oder Vorträge statt. Sechs speziell ausgestattete Schulungsräume, davon zwei mit einer Größe von 115 Quadratmetern, stehen im Nordbereich zusätzlich zur Verfügung, darunter auch zwei 10 Quadratmeter große Schreibwerkstätten. In den Schreibwerkstätten wird Studierenden 


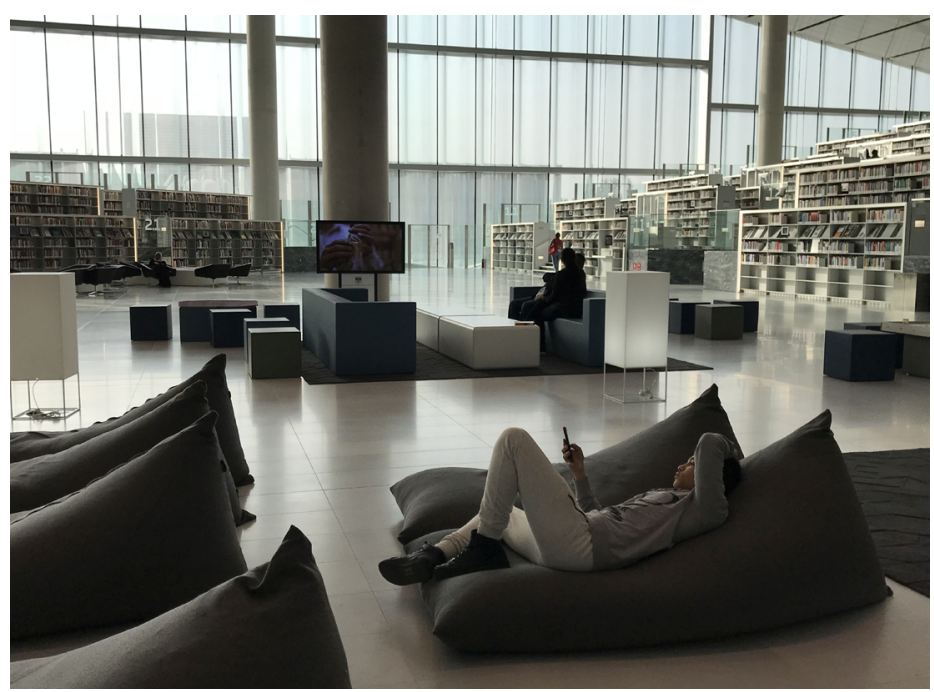

Abb. 10: Nationalbibliothek Katar, weiche Sitzmöbel auf der Plaza (Foto: C. Lux)

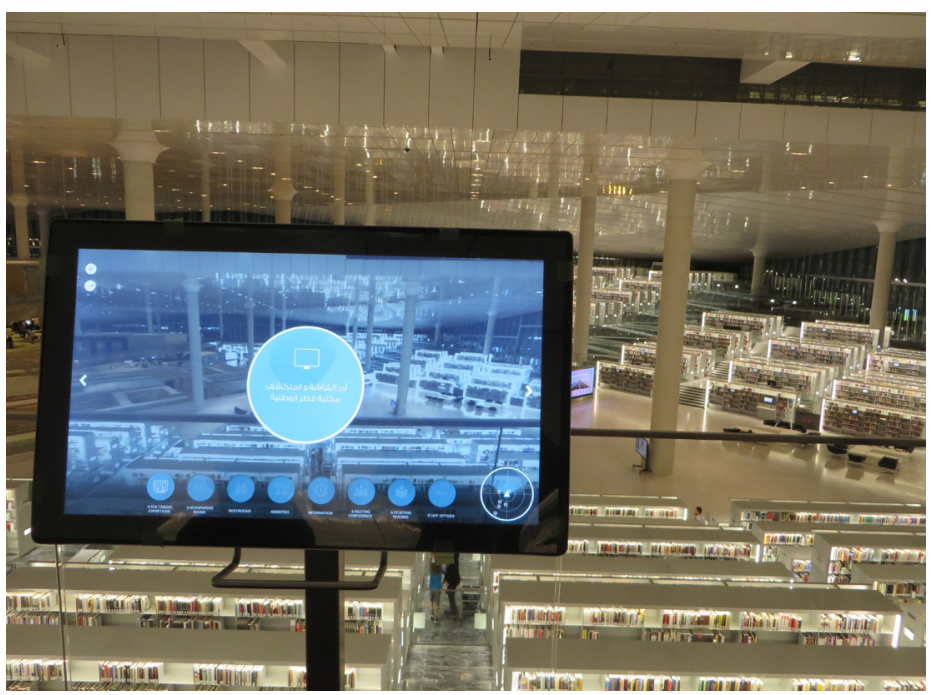

Abb. 11: Nationalbibliothek Katar, Informationsbildschirm (Foto: C. Lux)

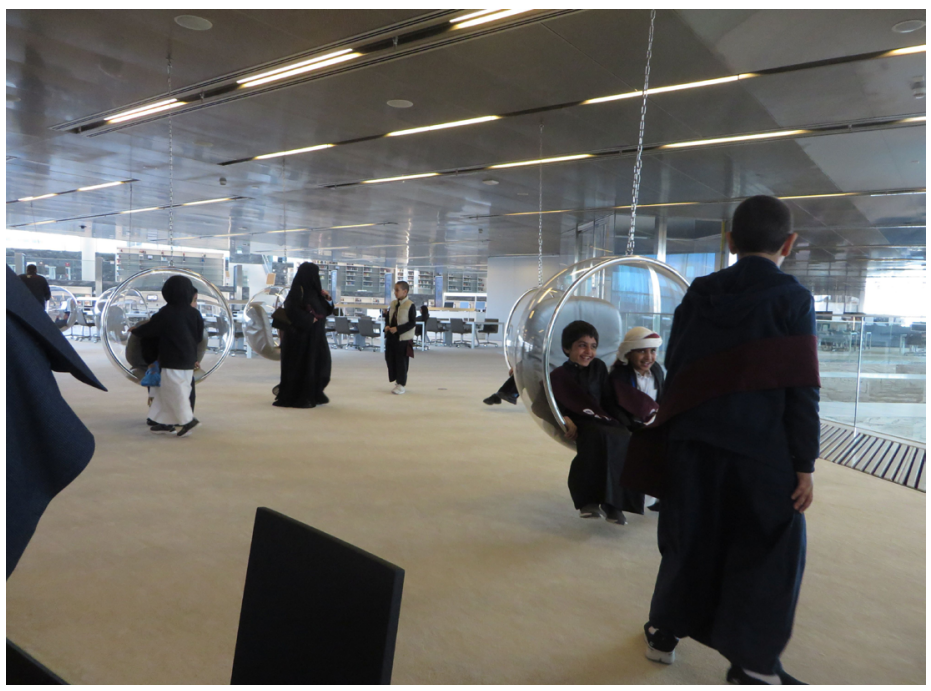

Abb. 12: Nationalbibliothek Katar, Kugelsitze zum Schaukeln (Foto: C. Lux) 


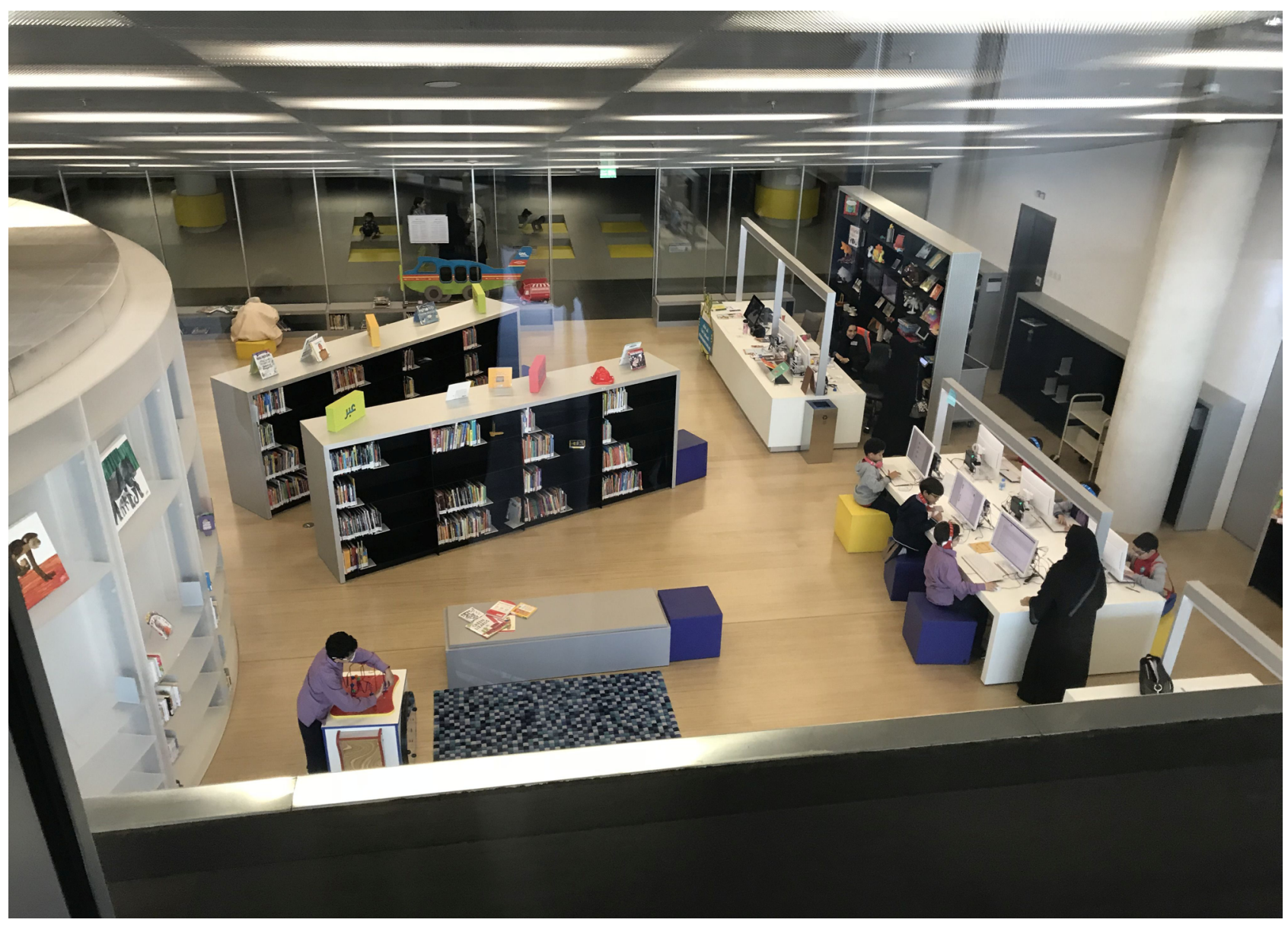

Abb. 13: Nationalbibliothek Katar, Kinderbibliothek (Foto: C. Lux)

bei dem korrekten Verfassen ihrer Abschlussarbeiten in Englisch oder Arabisch geholfen, und sie lernen die notwendigen Grundlagen des wissenschaftlichen Schreibens. Auch kreative Schreibkurse haben schon stattgefunden. In den Schulungsräumen finden regelmäßig Schulungen zur Informationskompetenz allgemein und spezielle Schulungen zur Nutzung bestimmter Datenbanken statt.

Auch an die ganz jungen Nutzerinnen und Nutzer wurde gedacht - eher ungewöhnlich für eine Nationalbibliothek! Im nördlichen Querriegel findet man unter den Terrassen die Kinderbibliothek, reich bestückt mit Kinderliteratur, Spielzeug und Medien, in der Mitte ein rundes Lesezelt für die Märchenstunde. Im Korridor davor befindet sich ein Bereich zum Klettern und Toben für den Bewegungsdrang der Kinder. Insgesamt verfügt die Bibliothek über einen Bestand von fast 100000 Kindermedien in verschiedenen Sprachen, und der Massenandrang in der Kinderbibliothek an jedem Wochenende zeigt, wie notwendig ein großer Buchbestand und große Räume sind. Gleich nach der Eröffnung wurden die angrenzenden Mitarbeiterräume mit integriert, da die überwältigende Nutzung der Kinderbibliothek keine andere Möglichkeit zuließ. Trotzdem ist dieser Bereich mit seinen 650 Quadratmetern viel zu klein für den Bedarf in Katar. Daran wird erst eine gezielte Modernisierung des öffentlichen Bibliothekswesens mit dem Ausbau von Kinderbibliotheken langfristig etwas ändern können.

Neben der Kinderbibliothek liegt das Restaurant, in dem sich Eltern aufhalten können, während die Kinder in der Kinderbibliothek oder in einer der Innovationsstationen eine Veranstaltung besuchen. Die Innovationsstationen sind der Maker-Space der Nationalbibliothek von Katar. In einem Raum kann man beispielsweise eigene Videos mit Unterstützung von Green Screen-Technik drehen, natürlich auch mit Anleitung. Nebenan steht ein 3D-Drucker, mit dem man sein eigenes Werkstück produzieren kann. Immer gibt es Hilfe, wenn man den 3D-Scanner oder die Geräte zur Digitalisierung alter Dias, Videos oder Tonbänder nutzen möchte. Auch eine elektronische Nähmaschine lädt ein, eigene Produkte zu verschönern. Ein spezieller Raum ist der Musik gewidmet, hier kann man Instrumente ausprobieren, seine eigene Komposition als 
CD produzieren oder Karaoke üben. Ein digitales Tonstudio unterstützt Kinder und Erwachsene bei ihren eigenen Kreationen.

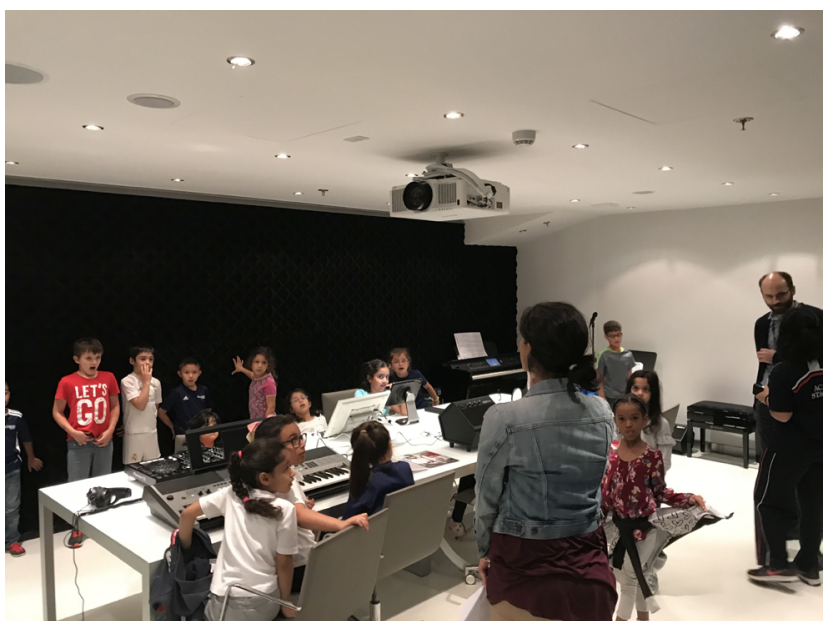

Abb. 14: Nationalbibliothek Katar, Kinder im Musiklabor (Foto: C. Lux)

Besonders beliebt sind die Virtual-Reality-Anwendungen (VR-Anwendungen). Hier gibt es ein starkes Interesse, solche Anwendungen auch selbst gestalten zu können. Spannend ist es, sich im Raum wie beim Tiefseetauchen zu bewegen und die bunten Fische durch die VR-Brille zu sehen.

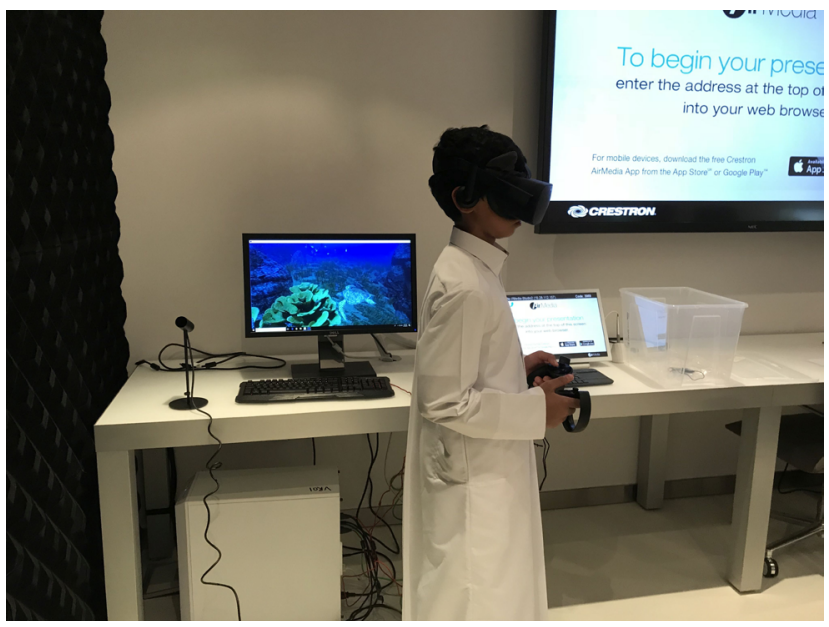

Abb. 15: Nationalbibliothek Katar, Innovationsstation für Virtual Reality, Tiefseetauchen (Foto: C. Lux)

Von den Räumen der Innovationsstationen aus führt der Gang auch zu den schön gestalteten Gebetsräumen, die in Katar in keinem Gebäude fehlen dürfen.

Auf der Westseite liegen die 15 Studierkabinen in der 24/7-Zone. Hier gibt es einen speziellen Ausgang, der eben- falls barrierefrei ist, und ursprünglich war geplant, dass Fakultätsangehörige und Studierende der Education City hier Tag und Nacht das Gebäude betreten und die Arbeitsplätze vor allem in Prüfungszeiten nutzen können. Die Öffnungszeiten betragen gegenwärtig für die ganze Bibliothek 10 Stunden, von 8.00 Uhr morgens bis 20.00 Uhr abends, bis auf Freitag, an dem die Bibliothek erst ab 16.00 Uhr geöffnet ist. Eine Erweiterung bis 22.00 Uhr abends ist gegenwärtig in der Planung. Leider ist das 24/7Konzept so kurz nach der Eröffnung noch nicht realisiert, obwohl es relativ einfach mit Sicherheitspersonal durchzuführen ist. Zumindest ist schon der Getränkeautomat für die Versorgung vorhanden. In diesem 24/7-Bereich sind auch die Angebote für sehbehinderte Nutzer und Nutzerinnen untergebracht, gleichgültig ob man noch Braille lesen möchte oder mit einer Sprachsoftware den Inhalt am Computer vorgelesen bekommt. Natürlich sind hier auch weitere offene Arbeitsplätze mit Blick auf den U-Bahnhof Qatar National Library an der Hauptstraße vorhanden.

\section{Der Online-Katalog, die Webseite, Datenbanken, Social- Media und Qatar Digital Library}

Der Onlinekatalog der Bibliothek enthält noch nicht alles, was in den letzten Jahren im Eiltempo angeschafft wurde, aber man hat schon einen guten Zugang zur englischsprachigen Literatur, da die Katalogdaten mit den Büchern eingekauft wurden. Nur für die arabisch- und anderssprachigen Bestände, die auf den Buchmessen in der Region gekauft wurden, müssen die Titelaufnahmen im Haus erstellt werden. Parallel zur Nationalbibliothek arbeitet auch die Dar-Al-Kutub-Bibliothek seit einigen Jahren ihre Bestände in den Katalog der Nationalbibliothek ein, so dass er sich zu einem Gesamtkatalog entwickelt. Gleichzeitig werden auch aus den lizensierten Datenbanken Titelaufnahmen eingespielt, damit das große elektronische Angebot vor allem im Bereich der Naturwissenschaften und Medizin genutzt wird. Dafür wurden an den Regalen im Freihandbereich iPads angebracht, mittels derer man nicht nur den genauen Standort des gesuchten Buchs abfragen kann, sondern auf denen man in Zukunft auch die elektronischen Medien $\mathrm{zu}$ dem betreffenden Fachgebiet angeboten bekommt, gerade wenn man etwas sucht, das man nicht im Regal vor sich findet.

Das digitale Angebot der Qatar National Library für ihre Nutzer ist seit Jahren außergewöhnlich. Mehr als 350000 E-Books, 70000 E-Journals und Hunderttau- 
sende Artikel sind im Angebot. Da das physische Bibliotheksgebäude erst im Herbst 2017 mit der Voraböffnung benutzbar wurde, wurde die Bibliothek bereits 2014 digital eröffnet. Jeder, der eine ID-Karte von Katar besitzt, kann eine Bibliothekskennung erhalten, mit der das digitale Angebot in ganz Katar nutzbar ist. Unter den mehr als 160 Datenbanken befinden sich wissenschaftliche Datenbanken von Springer und Elsevier, aber auch einige arabische Datenbanken und allgemein interessierende Datenbanken mit Musik, Videos und Romanen, wie z. B. die von Overdrive, die auch viele elektronische Bücher für Kinder und Jugendliche enthält. Dies hat dazu geführt, dass die Bibliothek schon lange vor der Eröffnung des neuen Gebäudes mehr als 20000 Mitglieder zählen konnte, die das digitale Angebot eifrig nutzten.

Da dieses Angebot vor allem über die Webseite propagiert wurde und die Anmeldungen nur darüber möglich waren, war der Ausbau der Webseite von Anfang an eine der wichtigsten Aufgaben. Aber in einem Land wie Katar ist eine Webseite allein nicht ausreichend. Nicht nur weil die Bevölkerung zu 99 Prozent Smartphones besitzt, sondern auch weil 99 Prozent der Bevölkerung Social Media nutzen, vorwiegend WhatsApp, Instagram, Twitter, Facebook. Keine Einrichtung kann in Katar überleben, die nicht intensiv in den Social Media präsent ist. Parallel dazu werden Ankündigungen und Informationen auf kleinen und großen Bildschirmen im Restaurant und an verschiedenen Stellen in der Bibliothek präsentiert, um auch die Nutzerinnen und Nutzer vor Ort wie auch das eigene Personal immer über die laufenden Ereignisse zu informieren. Daher ist es für eine Nationalbibliothek unabdingbar, eine große Abteilung für Öffentlichkeitsarbeit $\mathrm{zu}$ halten, die diese wichtigen Kommunikationsinstrumente bedient, laufend die neuen Aktivitäten ankündigt und anschließend wieder darüber twittert und informiert. Und dies natürlich in beiden Sprachen, Arabisch und Englisch.

Abgesehen von der Digitalisierung der eigenen historischen Werke hat die Nationalbibliothek ein großes Digitalisierungsprojekt mit der British Library abgeschlossen, das sich inzwischen in seiner dritten Phase befindet. Danach sollen die Archivdokumente der Kolonialzeit, wie sie in den Dokumenten des Indian Office Archives in der British Library liegen, digitalisiert werden, wenn sie einen Bezug zur Golfregion haben. Zusätzlich sollen die arabischen und islamischen Manuskripte aus London digitalisiert werden. Dafür richtete die British Library ab 2013 in ihrer Bibliothek ein ganzes Stockwerk ein, in dem für das Projekt mit der Qatar Foundation gearbeitet wird. Die relevanten Teile des Archivs wurden im Detail überprüft und digitalisiert. Außerdem wurde ein Portal aufgebaut, die Qatar Digital Library, in dem die Digitalisate mit ara-

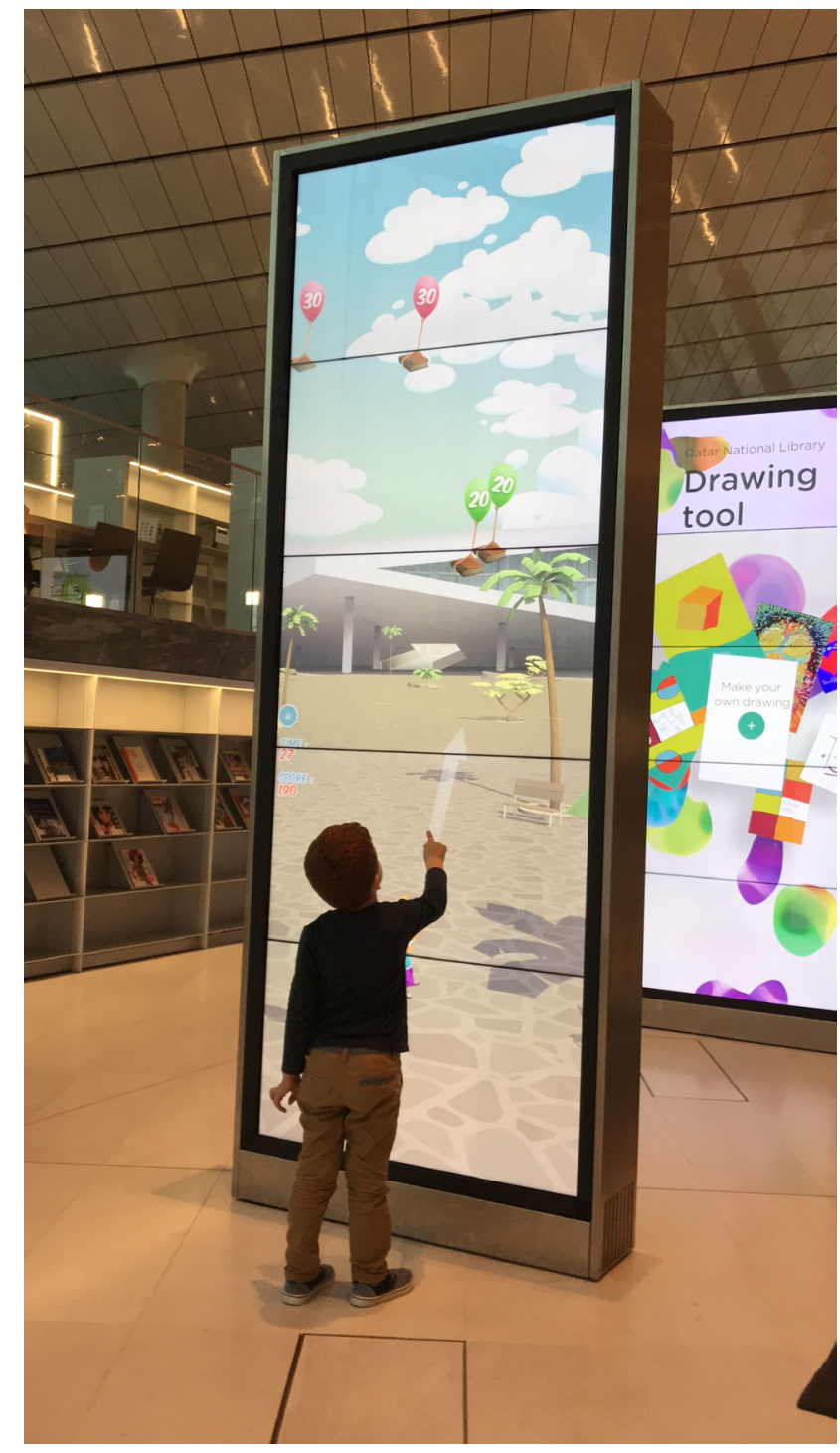

Abb. 16: Nationalbibliothek Katar, Infobildschirm zum Spielen und zur Werbung für die Veranstaltungen (Foto: C. Lux)

bischen und englischen Metadaten versehen weltweit frei verfügbar gestellt werden. Dieses Portal wird international stark genutzt, besonders auch von Ländern wie SaudiArabien und den Vereinigten Emiraten, die allerdings seit der Blockade gegen Qatar auch katarische Webseiten blockieren. Die hohe Wertigkeit der Qatar Digital Library liegt in der Tatsache, dass es bisher wenig digitalisierte historische Archivmaterialien über die Geschichte der Golfregion gibt. Bisher wurden zwar von verschiedenen Einrichtungen in der Region Kopien aus verschiedenen Archiven gesammelt, aber nur die freie Verfügbarkeit im Netz garantiert heute eine Vielzahl von Zugriffen und die systematische Forschung. ${ }^{5}$

5 Die Qatar Digital Library ist unter www.qdl.qa direkt erreichbar. 


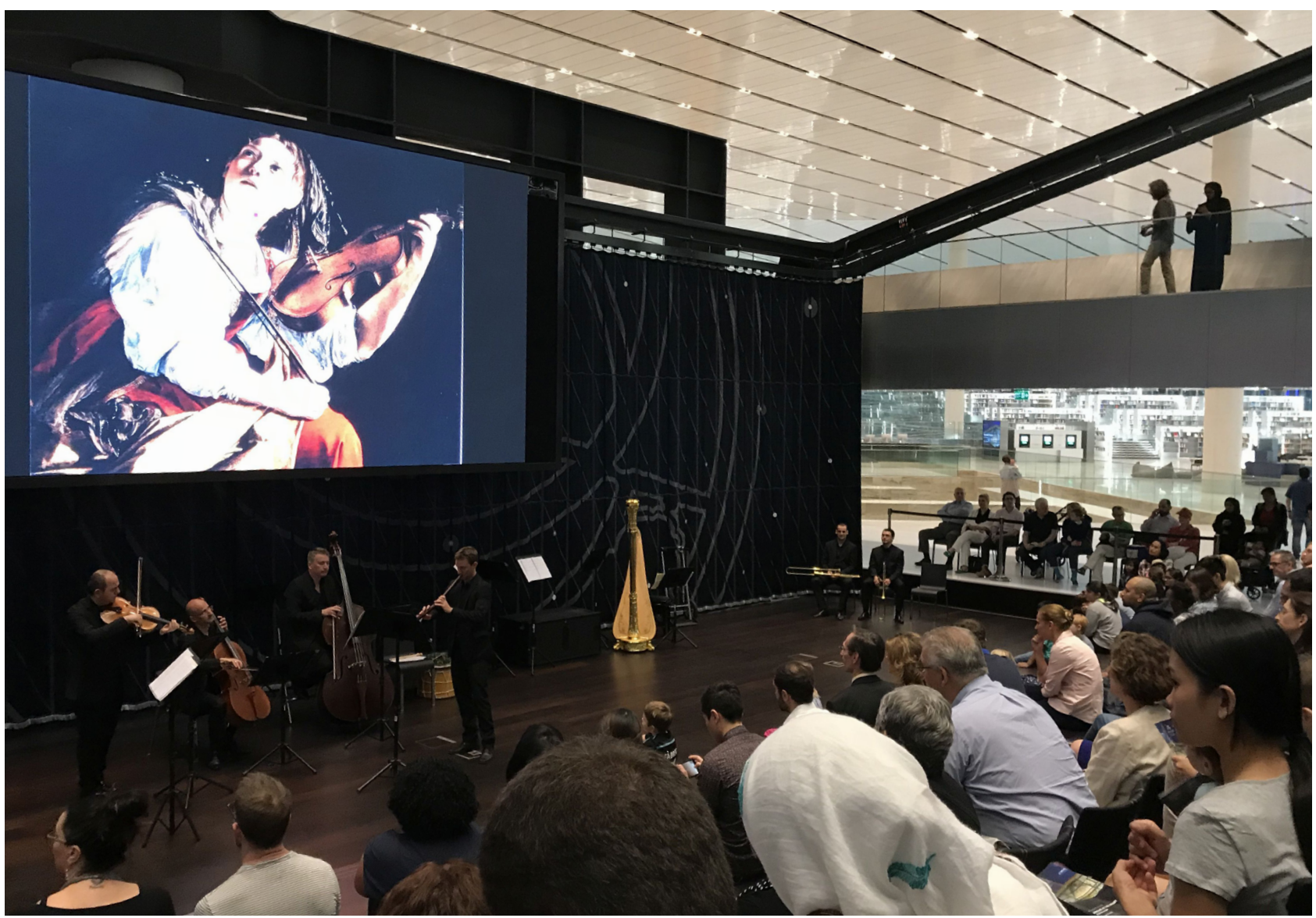

Abb. 17: Nationalbibliothek Katar, Barockensemble im Konzert (Foto: C. Lux)

Zusätzlich hat sich die Nationalbibliothek von Katar auch bei der World Digital Library ${ }^{6}$ engagiert und diese unterstützt, um das wichtigste nationale Erbe aller Staaten der Welt in einer Datei und in vielen Sprachen, darunter auch Arabisch, leicht zugänglich zu machen.

\section{Programmarbeit und Kunden}

Nahezu 60 bis 80 Aktivitäten werden jeden Monat in der neuen Nationalbibliothek angeboten, von Kinderveranstaltungen über Schulungskurse und Führungen durch die historische Sammlung bis hin zum Philharmonischen Orchester, das einmal im Monat in der Bibliothek spielt.

Ein männlicher Kollege hatte die besondere Idee eines Frauenstricktreffens, das wöchentlich eine engagierte Gruppe in die Bibliothek bringt, die auch noch Strickmusterbücher bespricht und ausleiht. Im Vordergrund steht aber die Kommunikation. Bei all diesen Angeboten ist es kein Wunder, dass sich die Besucherzahlen seit der Vor-

6 www.wdl.org (27.09.2018). aböffnung und noch mehr seit der offiziellen Eröffnung im April 2018 stetig weiterentwickeln. Von November 2017 bis August 2018 kamen 288408 Besucher in die neue Nationalbibliothek von Katar, das sind im Durchschnitt 1100 Besucher täglich. Dabei verzeichnet vor allem das Wochenende den stärksten Andrang. Die Anzahl der registrierten Nutzer stieg auf 85 030. Sie liehen 538167 Bücher und Medien (ohne E-Books und E-Journals) aus. Dabei ist zu beachten, dass die Anzahl der möglichen Ausleihen auf vier beschränkt wurde. Auch hier wird eine positive Veränderung mit Sicherheit weitere Zuwächse ermöglichen.

In einem Land, dessen öffentliches Bibliothekswesen bei dem Modernisierungsschub der letzten Jahre vergessen wurde, ist die neue Nationalbibliothek ein Beispiel für eine erfolgreiche Konzeption einer neuen, ansprechenden Bibliothek, die für jedermann ein dritter Ort mit geringen Zugangsschranken und vielfältigen Austauschs- und Kommunikationsmöglichkeiten sein kann. Es wird klar belegt, dass die Schönheit und Offenheit eines Gebäudes heute eine zentrale Rolle für die Akzeptanz eines öffentlichen Raumes durch die Gesellschaft spielen, und zwar gilt dies für alle Schichten der Gesellschaft. So lieben es 


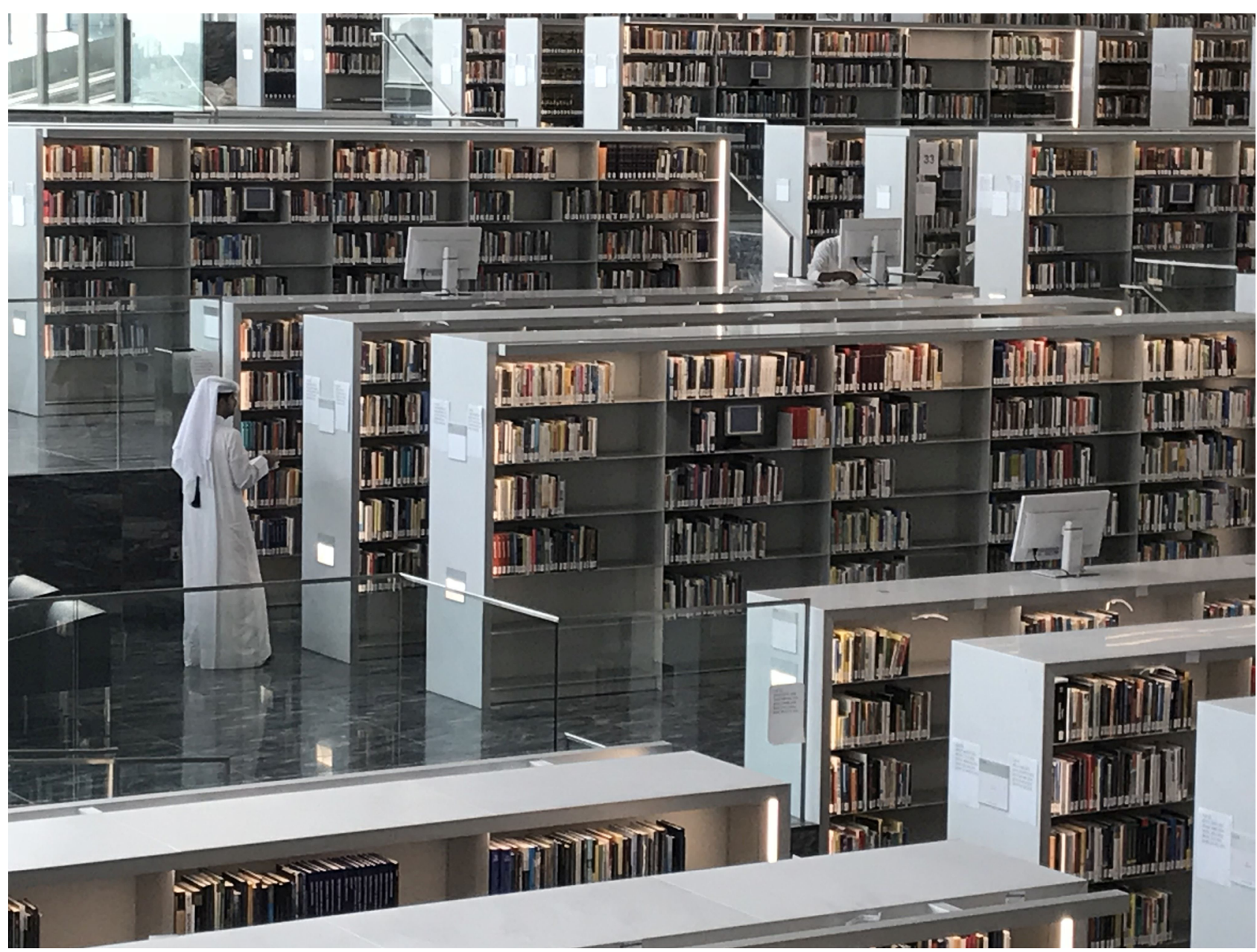

Abb. 18: Nationalbibliothek Katar, Katarer auf der Suche im Lesesaal (Foto: C. Lux)

die katarischen Studentinnen, umgeben von der Literatur ihres Faches, diese neue Bibliothek als ihren Lernort zu begreifen. Und auch eine Kinderbibliothek eröffnet Menschen, die sich niemals in eine Bibliothek getraut hätten, die Möglichkeit, den Raum wahrzunehmen und als ihren eigenen zu begreifen. Das Wichtigste aber ist die aktive Programmarbeit: Veranstaltungen, Schulungen, große Feste, Konzerte, die die Menschen ansprechen und ihnen die Freude an der Nutzung der Medien vermitteln.

Anmerkung: Die Verfasserin war von 2012 bis 2017 Direktorin der Nationalbibliothek von Katar. Sie hat die Bibliothek mit Personal, Technik, Sammlung und Dienstleistungen aufgebaut und die Anpassung des Baus an die bibliothekarischen Notwendigkeiten maßgeblich mitgestaltet.

\section{Autoreninformationen:}

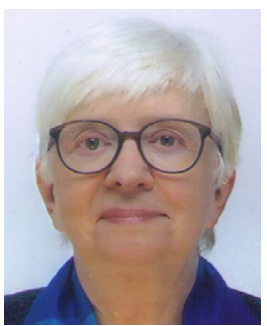

Prof. Dr. Claudia Lux

Humboldt Universität Berlin Institut für Bibliotheks- und Informationswissenschaften Dorotheenstr. 26 10117 Berlin claudia.lux@ibi.hu-berlin.de orcid.org/0000-0003-1655-4131 